\title{
VIABILIDADE DA APLICAÇÃO DE TEORIAS DE ENFERMAGEM: RELATO DE EXPERIENCIA NO ÂMBITO DA PESQUISA
}

\author{
Olga Maimoni Aguillar" \\ Isabel Amélia Costa Mendes**
}

AGUILLAR, O. M. \& MENDES, I. A. C. Viabilidade da aplicação de teorias de enfermagem: relato de experiência no âmbito da pesquisa. Rev. Esc. Enf. USP, São Paulo, 22 (n. especial): 47-52, jun. 1988.

Tecendo considerações sobre a importância das teorias de enfermagem para a promoção de uma abordagem unificada para a prática e para a pesquisa, as autoras apresentam sinopse do Modelo de Roy e relatam experiência de sua aplicação à pesquisa. Finalizam o artigo enfatizando a necessidade destas aplicações para que as teorias venham a servir de guias para a atuação do enfermeiro.

UNITERMOS: Teorias de enfermagem. Filosofia de enfermagem. Modelos teóricos.

Entendemos o advento das teorias de enfermagem como uma preocupação do profissional em imprimir orientação teórica que possibilite sistematizar a sua prática, desenvolver suas atividades apoiadas em um processo científico que the dê subsídios e the permita reflexão e avaliação, visando o aprimoramento de sua prática profissional. As teorias vêm nortear a pesquisa e favorecer o progresso do conhecimento na profissão. Observa-se então que a elaboração de teorias reflete a necessidade dos profissionais comporem um corpo de conhecimentos específicos da profissão, a fim de que a enfermagem possa vir a ser reconhecida como ciência.

A enfermagem tem demonstrado interesse em fenômenos que interferem na saúde e bem-estar dos indivíduos e da sociedade. Estamos de acordo com CHINN ${ }^{4}$ quando afirma que atualmente o desenvolvimento das teorias demonstra a vitalidade da enfermagem enquanto disciplina, cujo desenvolvimento futuro depende da articulação de direções que se desdobrem em educação, prática e pesquisa - estes desdobramentos estarão sempre relacionados ao desenvolvimento das teorias.

Entendemos as teorias como formas de representação da realidade. Dentre os modelos teóricos hoje existentes na enfermagem, percebe-se

\footnotetext{
- Professor Assistente do Departamento de Enfermagem Geral e Especlalizada da Escola de Enfermagem de Ribeirão Preto - USP

- Professor Assistente Doutor do Departamento de Enfermagem Geral e Especlallzada da Escola de Enfermagem de Ribeirăo Preto - USP.
} 
que os elementos básicos são comuns, variando apenas as suas relações. Os elementos básicos são: a pessoa, o ambiente, a saúde e a enfermagem. Diferentes autores colocam ênfase distinta em cada um desses elementos. estabelecendo relações peculiares a sua visão e intrepretação.

Os modelos conceituais na enfermagem têm se desenvolvido com muita rapidez nas últimas décadas e têm sido úteis para clarificar os seus conceitos e o seu processo de trabalho. Dentro do tema proposto, nos deteremos no modelo de Adaptação de Roy ${ }^{3,7,8,9,10 .}$

O modelo de adaptação de Roy ${ }^{9}, 10$ tem sido um dos mais amplamente estudados. Teve início nos fins da década de 60 quando Roy ainda era aluna do curso de graduação da Universidade da Califórnia, Los Angeles. Seus trabalhos foram influenciados pela sua formação em ciências sociais e refletem uma postura individual enquanto pessoa.

\section{CONCEITOS DO MODELO DE ROY}

Pessoa:

Roy descreve a pessoa como um sistema adaptativo com mecanismos inatos e adquiridos os quais the permitem competir com as mudanças internas e externas que the ocorrem. Classifica esses mecanismos como reguladores ou inatos, aqueles cuja resposta é automática e decorrente das atividades: neural, química e endócrina e mecanismos reconhecedores ou adquiridos, aqueles cuja resposta é dada por canais cognitivos/emotivos, isto é, comportamentos decorrentes do aprendizado, da experiência.

Os comportamentos decorrentes dos mecanismos reguladores e reconhecedores, podem ser efetivos ou inefetivos e são manfiestados por quatro modos de adaptação: a) fisiológico; b) auto-conceito; c) domínio de papéis; e d) interdependência.

\section{Ambiente:}

Para Roy, o ambiente inclui todas as circunstâncias que afetam o comportamento da pessoa, ou seja, estímulos internos ou externos que foram categorizados como: a) focal - aquele confrontado imediatamente pela pessoa e que ativa os mecanismos regulador e reconhecedor, criando uma necessidade em um dos quatro modos adaptativos; b) contextual — todos os outros estímulos presentes e que estejam influenciando o comportamento; e c) residual - todos os outros estímulos que possam influenciar a adaptação mas cujos efeitos nem sempre podem ser confirmados, como crenças e atitudes.

Neste conceito, Roy traz a definição de adaptação de HELSON ${ }^{5}$ (psicólogo fisiologista) a habilidade da pessoa em responder positivamente aos estímulos em uma dada situação e que esta habilidade é uma função do grau de mudança (demanda, exigência criada pelo estímulo) e as experiências anteriores. 
As respostas aos estímulos são chamadas adaptativas, quando promovem a integridade da pessoa em termos de sobrevivência, crescimento, reprodução e realização; que são os objetivos da adaptação. E são ineficazes as respostas que não contribuem para essa integridade.

\section{Saúde:}

Este conceito é decorrente do conceito de pessoa e ambiente e é definido como um estado e um processo de ser e tornar-se uma pessoa íntegra, ou seja, com alto potencial de realização. A pessoa é reconhecida como um sistema adaptativo holístico em constante crescimento e desenvolvimento, dentro de um ambiente em constantes mudanças, com um objetivo que é o de manter sua integridade através de respostas adaptativas.

Este conceito está em estágio de desenvolvimento e sua definição deve ser reavaliada e comprovada com o decorrer de sua utilização na prática.

\section{Enfermagem:}

O objetivo da enfermagem dentro do modelo, é promover a adaptação da pessoa em situações de saúde e doença, enquanto indivíduo, família ou comunidade.

Para isso, Roy propõe o processo de enfermagem com os seguintes passos, para direcionarem as ações do profissional de enfermagem:

1) Avaliação dos comportamentos.

- Através da observação, mensuração, relatos ou entrevistas.

- Realiza-se a categorização dos comportamentos nos quatro modos: fisiológico, auto-conceito, domínio de papéis e interdependência.

- Classifica-se o comportamento em: adaptativo ou inefetivo.

2) Avaliação dos estímulos.

- Através da observação, mensuração, relatos e entrevistas.

- Realiza-se a categorização dos estímulos (juntamente com o pa. ciente): a) focal, b) contextual e c) residual que estão afetando a resposta comportamental.

3) Diagnóstico de Enfermagem.

Através da avaliação do comportamento e dos estímulos causadores, é estabelecido o diagnóstico de enfermagem em relação ao problema adaptativo - interpretação dos dados coletados.

4) Estabelecimento de objetivos.

- O objetivo geral da enfermagem é a manutenção do comportamento adaptativo e tornar os comportamentos inefetivos em adaptativos.

- Os objetivos específicos são estabelecidos para cada situação e deve prever espaço de tempo.

- De preferência ele deve ser estabelecido juntamente com o paciente. 
5) Intervenção de Enfermagem.

- Neste passo, estabelece-se quais ações devem ser realizadas para alcançar os objetivos propostos a curto, médio e longo prazo. Aqui são estabelecidas prioridades em relação ao que deve ser abordado em primeira instância.

6) Avaliação.

O último passo, envolve a comparação entre os objetivos estabelecidos e os resultados alcançados, para se observar o sucesso ou não do plano.

\section{EXPERIENCIA DE APLICAÇÃO DO MODELO A PESQUISA}

Nossa experiência com a utilização do modelo em questão, está restrito ao âmbito da pesquisa. Passaremos a relatar a sua utilização por AGUILLAR \& ANGERAMI ${ }^{2}$ no trabalho: "Contribuição ao estudo do processo de adaptação da pessoa laringectomizada", que teve como objetivos: a) identificar situações nas quais os pacientes laringectomizados apresentam problemas de adaptação e b) propor intervenções de enfermagem para promover a adaptação do paciente.

A população constou de pacientes atendidos no Hospital das Clínicas de Ribeirão Preto e a amostra constitui-se de 17 pacientes que sofreram laringectomia total por serem portadores de câncer de laringe.

Para cada paciente foi elaborado um estudo de caso com dados obtidos da observação do seu comportamento, do prontuário e de entrevistas semanais por um período médio de 4-6 meses e nos retornos após 6 meses e um ano após a alta. Estas entrevistas tinham como finalidade 0 aprendizado da voz esofágica e a coleta de dados relacionados ao processo de adaptaçã̃o do paciente.

O desenvolvimento do referido estudo obedeceu as seguintes etapas:

a) Estudo de caso.

b) Identificação dos comportamentos e respectivos estímulos.

c) Avaliação dos comportamentos (diagnóstico de enfermagem).

d) Intervenções de enfermagem.

e) Localização no "continnuum" saúde-doença.

Em relação ao primeiro objetivo, concluiu-se que são geradores de problemas adaptativos as situações que se seguem:

1) Conhecimento do diagnóstico.

2) Trauma cirúrgico.

3) Tratamentos coadjuvantes.

4) Instalação da traqueostomia.

5) Afasia.

6) Situação sócio-econômica.

7) Retorno ao trabalho.

8) Lazer.

9) Relacionamento sexual. 
Em relação ao segundo objetivo, a orientação para o aprendizado da voz esofágica, pré-estabelecida, tendo em vista a afasia destes pacientes, foi a motivação para o contacto inicial. As demais intervenções ocorreram na medida em que os problemas se apresentaram e constituíram-se de: orientações, demonstrações, encaminhamentos e outros.

Através da metodologia, foi possivel ordenar os dados e identificar os probelmas adaptativos dos pacientes laringectomizados; entretanto, algumas dificuldades foram encontradas, na avaliação dos comportamentos em adaptativos ou inefetivos, para tanto, foram convidados três juízes que colaboraram nessa avaliação.

Concluiu-se que um estímulo pode gerar comportamentos em mais de um modo, o que muitas vezes dificulta a sua identificação. Os comportamentos inefetivos do modo fisiológico são mais facilmente identificáveis e foram semelhantes para diferentes pacientes. Entretanto, os do modo do auto-conceito, domínio de papéis e interdependência, são de complexa identificação, sua freqüência é alta e os comportamentos são diversos frente a estímulos semelhantes.

Neste estudo ${ }^{1,2}$, não foi possível determinar se existe prioridade em relação aos problemas adaptativos identificados. Nem mesmo foi possível a autora afirmar que o problema da perda da voz seja o mais importante para todo paciente laringectomizado.

Utilizando o modelo conceitual de Roy, em estudo com mulheres sexualmente violentadas, LIMANDRI ${ }^{6}$ aponta algumas dificuldades encontradas na aplicação do modelo. Considera impossivel separar os modos do auto-conceito, domínio de papéis e interdependência como entidades distintas; afirma que o modelo é mais um constructo teórico do que uma categorização empírica, oriunda da avaliação do paciente. $\mathrm{Na}$ opinião da autora os modos de adaptação não constituem variáveis independentes, como sugere o modelo. Considera também muito obscuro ainda o relacio. namento entre os mecanismos reguladores e reconhecedores.

Alerta para o fato de que ao sobrepor o mecanismo regulador ao modo fisiológico, Roy confundiu a distinção entre os dois.

A autora ${ }^{6}$ propôs modificações à teoria de Roy a partir do referido estudo realizado com mulheres sexualmente violentadas, modificações estas extraídas dos dados empíricos provenientes de pesquisa e prática. Salienta a essencialidade deste processo para uma perfeita ligação entre o desenvolvimento da teoria e a expansão do conhecimento da enfermagem enquanto disciplina.

Finalizando, gostaríamos de colocar que acreditamos que só através da aplicação dos modelos teóricos à pesquisa e à prática é que poderão ser determinadas suas forças, suas fraquezas, sugestões para sua modificação, enfim, sua testagem, de modo que surjam condições propícias para o fortalecimento das estruturas teóricas como guias para a atuação do enfermeiro: na prática, no ensino e na pesquisa. 
AGUILLAR, O. M. \& MENDES, I. A. C. Applicability of nursing theories: Rev. Esc. Enf. USP,

São Paulo, 22 (special issue): 47-52, June 1988.

Discussing the importance of nursing theories for the promotion of a unified approach to practice and teaching, the authors present a synopsis of the Roy Model and report an experience with its application to research. They conclude the article by emphasizing the need for more of these applications so that theories may come to serve as guidelines for the nurse's performance.

UNITERMS: Philosophy, nursing. Nursing theory. Models, theoretical.

\section{REFERENCIAS BIBLIOGRAFICAS}

1 - AGUILLAR, O. M. \& ANGERAMI, E. L. S. Avaliação sistematizada na reabilitação da voz de pacientes laringectomizados. Rev. Esc. Enf. USP. São Paulo, 17 (1):47-60, abr. 1983.

2 - Contribuição ao estudo do processo de adaptação da pessoa laringectomizada. Rev. Bras. Enf., Brasilia, 37 (3/4):174-81, jul./dez. 1984.

3 - ANDREWS, H. A. \& ROY, C. Essentials of the Roy adaptation model. London, Prentice-Hall, 1986.

4 - CHINN, P. Nursing theory development: where we have been and where we are. going. IN: CHASKA, N. L. The nursing profession: a time to speak. New York, McGraw-Hill Book, 1983. cap. 29, p. 394-405.

5 - HELSON, H. Adaptation: level theory. New York, Harper \& How, 1964.

6 - LIMANDRI, B. J. Research and practice with abused women: use of the Roy adaptation model as an explanatory framework. A. N. S., Germantou'n, 8 (4):52-62, July, 1986.

7 - RIEHL, J. P. \& ROY, C. Conceptual models for nursing practice. New York, PrenticeHall, 1974.

8 - ROY, C. \& ROBERTS, S. L. Theory construction in nursing: an adaptation model. London, Prentice-Hall, 1981.

9 - ROY, C. Adaptation: a conceptual framework for nursing. Nurs. Outlook, New York, 18 (3):42-5, Mar. 1970.

10 - Adaptation: a basis for nursing practice. Nurs. Outlook, New York, 19 (4):254-7, Mar. 1971. 